\title{
LAS ASOCIACIONES DE INMIGRANTES AFRICANOS Organización, proyección y actuaciones
}

\author{
ASSOCIATIONS OF AFRICAN IMMIGRANTS \\ Organization, projection and activities
}

JoRDI GARRETA BoCHACA jgarreta@geosoc.udl.cat

Universidad de Lleida. España

Núria Llevot Calvet nllevot@pip.udl.cat

Universidad de Lleida. España

\begin{abstract}
RESUMEN
El asociacionismo de personas de origen extranjero ha crecido de forma notable desde los años $90 \mathrm{y}$, especialmente, desde los 2000, influenciado por los intereses de estas personas pero también por el contexto social, político e institucional que lo ha favorecido y condicionado. Este trabajo presenta los resultados de una encuesta realizada a 206 representantes de asociaciones de inmigrantes de origen africano de las comunidades autónomas de Cataluña, Valencia y Navarra. Nuestro objetivo ha consistido en clasificar las asociaciones, estudiar su organización y funcionamiento y conocer sus ámbitos de actuación. Entre otras cuestiones, hemos observado que este asociacionismo es joven y no responde a una única forma organizativa, sino que, en función de dinámicas internas y externas, intereses de los asociados y años de vida de cada asociación, adquiere diferentes estructuras y estrategias.
\end{abstract}

Palabras Clave

Inmigración; Asociacionismo; Inmigrantes de origen africano; Organización;Actividades.

\begin{abstract}
Associations for foreign-origin people have grown notably since the nineteen-nineties, and more so in the first decade of the $21^{\text {st }}$ century. This growth has been influenced by the interests of their members but also by the social, political and institutional context that has favoured, while also conditioning, them. The work presented here contains the results of a survey carried out among 206 representatives of associations of African-origin immigrants in the Catalonia, Valencia and Navarre regions, with the aim of defining the profile of these associations, their organisation, working and activities. Among the questions dealt with, we see how this associational activity is still relatively new. It has no common organizational structure, but rather the structures and strategies that each association acquires depend on its internal and external dynamics, its members' interests and its age.
\end{abstract}

\section{KEYWORDS}

Immigration; Establishment of Associations; African-Origin Immigrants; Organisation;Activities. 


\section{INTRODUCCIÓN}

En 1830, A. Tocqueville, estudiando el pluralismo norteamericano, destacaba la importancia del asociacionismo - político, social, religioso y económico- como una forma de representación de los intereses de los diferentes grupos existentes en una sociedad y también como un mecanismo de información y de participación. La literatura sobre la inmigración y los procesos de incorporación de los inmigrantes a las sociedades de destino a menudo ha enfatizado la importancia de la organización y la construcción de redes como elementos clave de la movilización social y política. Otro elemento que ha sido objeto de estudio ha sido la influencia del entorno en estas organizaciones.

El hecho de que existan pocos estudios sobre la organización y el funcionamiento de las asociaciones de extranjeros de origen africano en España ha despertado nuestro interés y nos ha llevado a analizar la estructura y la organización interna y externa de las asociaciones de este perfil, y a relacionarlas con los objetivos y las actividades que realizan. Nuestro punto de partida es la creencia de que existen estructuras organizativas y desarrollos diferentes, influidos por el entorno y por las dinámicas internas que condicionan el día a día (objetivos y actividades) de las asociaciones. Además, al concretar lo que pretenden hacer y lo que efectivamente hacen, creemos que el citado asociacionismo tiene un escaso interés político y se centra prioritariamente en lo sociocultural. A continuación, una vez revisada la literatura científica sobre asociacionismo e inmigración, nos centraremos en los resultados obtenidos a través de una encuesta a representantes de asociaciones formadas por extranjeros procedentes de África, lo que nos ha facilitado acercarnos a las asociaciones y conocer su organización y proyección, su evolución, y sus objetivos y actividades, y nos ha permitido, a partir de variables relacionadas con la organización y la proyección externa de las asociaciones, establecer una clasificación que las comprenda y tipifique.

\section{INMIGRACIÓN Y ASOCIACIONISMO}

Para R. Kastoryano (1994), la capacidad de negociación de un grupo o comunidad depende de su organización, del grado de participación de sus miembros y del impacto que tienen en su contexto social. No obstante, las políticas de gestión de la diversidad cultural tienen diferentes formas de percibir, potenciar y/o desincentivar la organización y creación de redes entre las personas de origen inmigrado, puesto que, aunque un entorno (social, institucional, político, etc.) favorecedor del asociacionismo

Este artículo se inscribe dentro del proyecto de investigación "Asociacionismo e inmigración africana: funciones latentes y manifiestas", financiado por el Ministerio de Ciencia e Innovación (CSO2008- 01122/SOCI) 
representa una mayor posibilidad de florecimiento y desarrollo de estas organizaciones, no siempre su "no potenciación" significa que no aparezcan y crezcan espontáneamente ${ }^{1}$. El trabajo de M. Fennema y J. N. Tillie (2004), en el que se compara Ámsterdam, Lieja y Zúrich, muestra que la densidad de las organizaciones étnicas es mayor, así como que la gama de actividades que desarrollan es más amplia en las dos primeras ciudades que en Zúrich, cuyas autoridades no han facilitado la formación de estas organizaciones ${ }^{2}$. En todos estos casos, podríamos hallar políticas que han animado su desarrollo al valorar el papel de lo comunitario, políticas que lo han hecho sin pretenderlo o, incluso, aquellas que lo han dificultado, con mayor o menor éxito y con más o menos voluntad, ya que han creído que la comunidad no debía mediar entre la nación y la ciudadanía.

En España, la evolución de la inmigración ha comportado la sedentarización de una parte de los inmigrantes y la aparición de organizaciones (culturales, religiosas, etc.) que los representan. Una de las manifestaciones de este fenómeno ha sido la creación, voluntaria o inducida, de asociaciones formadas y presididas por extranjeros que han desempeñado diferentes papeles según el momento, el contexto, su grado de consolidación, etc ${ }^{3}$. De hecho, existe una multiplicidad de entidades de solidaridad implicadas en la provisión de servicios a la población inmigrante ${ }^{4}$, lo cual no es irrelevante dado que, a pesar de poder compartir finalidades y objetivos, representan realidades muy diferenciadas; por ejemplo, en lo referente a las estrategias de intervención, a los retos que deben afrontarse, etc. (Morell 2005).

El Colectivo IOE (1999) considera que, entre los factores que favorecen el desarrollo del movimiento asociativo de personas extranjeras en España, destaca el refuerzo (no siempre exento de conflictos) proporcionado por determinadas organizaciones no gubernamentales y sindicatos, así como la capacidad que demuestran para captar fondos y gestionar recursos. G. Martín (2004), pone de manifiesto cómo los poderes públicos tienen la voluntad de financiar a las asociaciones con cargo a fondos públicos y de potenciar espacios de participación a escala nacional y autonómica. Este desarrollo del asociacionismo, que en sus inicios apostaba por la ayuda mutua y la solidaridad, se fue transformando durante los años noventa en acciones más reivindicativas (Aubarell 2003). A pesar de que se observa un incremento de las asociaciones

\footnotetext{
${ }^{1}$ Como indica K. Pilati (2012), existen otras posibilidades para las organizaciones de inmigrantes de autoorganizarse y de participar que las previstas por el contexto.

${ }^{2}$ Por otro lado, hay estudios que sugieren que, en función del reconocimiento o no reconocimiento que hacen las instituciones, se desarrollan los diferentes tipos de organización, así como las estructuras organizativas [Diani (2000) citado por Pilati (2012)].

${ }^{3}$ Castles, S. y M. J. Miller (1994) indicaban que las asociaciones voluntarias de inmigrantes son una manifestación necesaria de su asentamiento y que, lejos de dificultar la integración de los grupos minoritarios, facilitan la negociación de su participación y su integración efectiva.

${ }^{4}$ Y clasificaciones de las mismas en España: Colectivo IOE (1994), J. Casey (1995), R. Crespo (1997), A. Morell (2005), J. Pont (2005), etc.
} 
y su consolidación, estas se hallan condicionadas por el entorno institucional y social, así como por dinámicas internas $\mathrm{y}$, por tanto, experimentan constantes procesos de reestructuración, cuando no de fisión y de fusión (Garreta 1998).

En España no se han hecho muchos estudios sobre la organización y el funcionamiento de las asociaciones ( $\mathrm{y}$, especialmente, de aquellas formadas por individuos procedentes de África). Para C. Gómez Gil (2006), la debilidad del movimiento asociativo de las personas de origen extranjero, sobre todo de los no comunitarios, su heterogeneidad y su fragmentación social son fenómenos habituales en España e inherentes a los procesos de asentamiento y reconocimiento legal. Su trabajo, centrado en Alicante, le lleva a afirmar que las asociaciones de inmigrantes extracomunitarios son: débiles, jóvenes, con fines y modelos organizativos diversos, con medios precarios y objetivos limitados. Todo ello es el resultado de la evolución y la situación de los inmigrantes, así como de la respuesta del entorno, que no ha animado suficientemente a su creación. Por su parte, C. Simó et al. (2005) también afirman que el asociacionismo en la Comunidad Valenciana es un fenómeno reciente, que sigue conformándose, marcado por la desigualdad organizativa y la escasa coordinación; aunque, eso sí, los autores detectan algunas organizaciones bastante consolidadas.

El trabajo de R. Aparicio y A. Tornos (2010), en el que nos ofrecen una visión de conjunto de las asociaciones de inmigrantes en España, considera relevante tener en cuenta el tamaño de la asociación en las lógicas de funcionamiento: las mayores (en número de socios) se estructuran diferenciando funciones, ajustando sus proyectos o sus recursos y programando actividades, mientras que las menores (que aceptan su papel) hacen menos diferenciaciones y también ajustan sus proyectos y actividades. Por otro lado, las menores que no aceptan su situación y las grandes que han crecido poco desde su creación crean estructuras ficticias y proyectos poco proporcionados en relación a su capacidad. El estudio apunta que la efectividad de las asociaciones en el cumplimiento de sus fines y en la realización de proyectos se ha relacionado a menudo con la organización interna ${ }^{5} \mathrm{y}$ las formas de liderazgo propias de cada una de ellas.

En general, las investigaciones españolas indican que, en el proceso de asentamiento de los inmigrantes en la sociedad de acogida, el asociacionismo debería: permitirles superar el aislamiento social, fomentar la sociabilidad y el intercambio de experiencias, y ayudarles a encontrar referentes válidos que les permitan una efectiva integración en la sociedad (Aubarell 2003), así como negociar su acomodación en el nuevo entorno y actuar como constructores y reconstructores de la identidad colectiva (Veredas 1999) y, al mismo tiempo, dotarse de un marco de pertenencia que les proporcione seguridad (Lluch 2000). Para R. Sipi (2000), la asociación representa el espacio en el que se destruye el aislamiento social que comporta vivir en las coordenadas clásicas de la inmigración.

\footnotetext{
${ }^{5} \mathrm{Al}$ referirse a la organización, apunta que, de las encuestas que realizaron, obtuvieron que el $99 \%$ tiene presidente y secretario; el $74 \%$, consejo directivo; el $93 \%$ celebra asambleas generales; el $65 \%$ tiene comités para tareas específicas, y el $95 \%$ tiene los estatutos escritos.
} 
Pero también es cierto que el papel de las asociaciones ha sido presentado por los investigadores como ambivalente, ya que, al mismo tiempo que facilitan la integración en la sociedad receptora, en la medida en que comportan un replegamiento étnico y contribuyen a reproducir los esquemas propios de la sociedad de origen, también la pueden dificultar. Los trabajos de J. Garreta (1998 y 2003) indican que las asociaciones, entendidas como una forma de vertebrar una minoría y defender sus intereses, es decir, como una base para la acción colectiva, pueden incidir favorablemente en el proceso de integración social, aunque para ello sea necesario evitar que institucionalicen estructuras paralelas que podrían derivar en una guetización.

\section{Las asociaciones de inmigrantes africanos en Cataluña, Comunidad ValenCIANA y NaVARRA}

A partir de este momento, nos centraremos en los resultados obtenidos en una encuesta a representantes de asociaciones formadas principalmente por personas de origen extranjero procedentes de África ${ }^{6}$, concretamente a una muestra formada por 206 representantes de asociaciones obtenida a través de un intenso barrido en las comunidades autónomas de Cataluña, Valencia y Navarra ${ }^{7}$. El objetivo del cuestionario, que empíricamente se cumplimentó mediante entrevista telefónica, era confeccionar un perfil del representante entrevistado $y$, sobre todo, de la asociación a la que representaba; de ahí que un gran número de preguntas versaran sobre la asociación, sus actividades, su área de influencia, sus necesidades, la coordinación que mantenían, etc. Desde ahora nos centraremos en las cuestiones que definen cómo son y en qué trabajan estas asociaciones. Concretamente, realizaremos un retrato de las asociaciones, de su organización y proyección, de sus actividades, y nos aproximaremos a ellas a partir de una tipología que pretende poner de manifiesto las diferencias existentes entre ellas y su diferente grado de consolidación.

\section{Las asociaciones de inmigrantes africanos: perfil básico}

Para definir el nacimiento de las asociaciones, se preguntó el año de creación y el funcionamiento informal antes de esta fecha. Como observamos en la tabla siguiente,

\footnotetext{
${ }^{6}$ El criterio para la selección de las asociaciones en las que realizamos encuestas y entrevistas en profundidad era que se tratase de aquellas que están formadas principalmente por personas de origen africano, es decir, aunque pudiera haber asociados de algún otro origen, los africanos debían ser más de las tres cuartas partes del total.

${ }^{7}$ Para conocer en profundidad la metodología de investigación es imprescindible consultar la 'Introducción Metodológica' al monográfico "Asociacionismo e inmigración" de la Revista Internacional de Sociología donde se encuentra este artículo.
} 
aunque hallamos asociaciones creadas antes y durante la década de los 90 , un número importante de ellas aparece durante el año 2000 y siguientes. También podemos observar que las asociaciones de la Comunidad Valenciana y Navarra son más jóvenes en comparación con las de Cataluña, lo cual se corresponde con la llegada de población extranjera de origen africano a cada comunidad autónoma y de las diversas dinámicas de consolidación del asociacionismo (véase Garreta 2003, 2009 y 2012; Lacomba 2004; Simó et al. 2005; Gómez Gil 2006 y Mata 2011).

El año de creación no quiere decir que con anterioridad las asociaciones no funcionaran informalmente. Por ello, se preguntó sobre esta cuestión y los resultados indicaron que una tercera parte de las asociaciones tuvo un funcionamiento previo antes de su creación o, mejor dicho, antes de su constitución más formal. Este funcionamiento previo es más frecuente en aquellas asociaciones que se encuentran inscritas en registros (el $41,7 \%$ frente a un $24,5 \%$ ).

El hecho de que estén constituidas y que mayoritariamente digan que disponen de estatutos ${ }^{8}$ no quiere decir que estén registradas oficialmente 0 en otro tipo de directorio -es decir, la respuesta dada se entiende en sentido amplio-, como indica el hecho de que un $50 \%$ de las asociaciones a las que realizamos la entrevista sí estaban registradas. Más concretamente, este registro es mayor en la Comunidad Valenciana (81,5\%) que en Navarra (42,9\%) o en Cataluña (38,4\%). Entre las inscritas en un registro, el $30,1 \%$ dice que se han registrado a nivel autonómico; el 16,5\%, en la Administración estatal; el $37,9 \%$, en el directorio de La Caixa; el 17,5\%, en ayuntamientos, y el 2,9\%, en otros bancos y cajas. Como puede verse, el número de asociaciones registradas es bajo.

Otra de las cuestiones definitorias de las asociaciones es su representatividad. En este punto, dado que somos conocedores de que existen personas que no se sienten representadas por las asociaciones (Garreta 1998), otras que no se vinculan a ellas por otros motivos [veáse Lacomba y Giner (2013)] y que algunos de nuestros interlocutores querían "darse importancia" a través del número de personas a las que dicen representar, realizamos dos preguntas que nos permitieron acercarnos a la representatividad real de las asociaciones. Una de las cuestiones se centraba en conocer el número de asociados y, la otra, el número de beneficiarios, es decir, el número de personas que reciben algún beneficio de la existencia de la asociación aunque no estén asociadas a ella [lo que J. Lacomba y J. Giner (op. cit.) han Ilamado "usuarios que no participan"].

Como se indica en la tabla 2, la media de asociados es de 153,4 (de los cuales el $68,8 \%$ son hombres y el $31,2 \%$ mujeres ${ }^{9}$ ). Concretando más, la edad de los asociados se situaría, principalmente, entre los 25 y 45 años. De hecho, el 7,68\% serían menores de 25 años; el 39,04\% tendría entre 25 y 35 años; el 40,02\%, entre 36 y 45, y el 23,27\%

\footnotetext{
${ }^{8}$ Prácticamente todas ellas (menos el 0,5\%) tienen los estatutos de la asociación aprobados.

${ }^{9}$ Las asociaciones a las que hemos realizado la encuesta presentan el siguiente perfil en cuanto a género de los asociados: el 78,5\% está formada solo, o principalmente, por hombres; el 8,73\% es mixta y el $12,77 \%$ está formada solo, o principalmente, por mujeres.
} 
Tabla 1.

Año de creación de la asociación

\begin{tabular}{l|c|ccc|ccc}
\hline & & \multicolumn{3}{|c|}{ COMUNIDADAUTÓNOMA } & \multicolumn{3}{c}{ ZONAORIGENASOCIADOS $^{*}$} \\
\cline { 2 - 8 } & TOTAL & Cataluña & $\begin{array}{c}\text { Valenciana } \\
\text { Antes de } 1990\end{array}$ & Navarra & $\begin{array}{c}\text { Norte } \\
\text { África }\end{array}$ & Mixta & Subsáhara \\
\hline $1990-1995$ & $11,2 \%$ & $12,3 \%$ & $3,7 \%$ & $28,6 \%$ & $8,1 \%$ & $22,1 \%$ & $3,9 \%$ \\
\hline $1996-2000$ & $20,9 \%$ & $21 \%$ & $24,1 \%$ & $7,1 \%$ & $27,4 \%$ & $16,2 \%$ & $19,7 \%$ \\
\hline $2001-2005$ & $38,3 \%$ & $36,2 \%$ & $46,3 \%$ & $28,6 \%$ & $38,7 \%$ & $23,5 \%$ & $51,3 \%$ \\
\hline $2006-2009$ & $23,3 \%$ & $21,7 \%$ & $24,1 \%$ & $35,7 \%$ & $25,8 \%$ & $29,4 \%$ & $15,8 \%$ \\
\hline Total & $100 \%$ & $100 \%$ & $100 \%$ & $100 \%$ & $100 \%$ & $100 \%$ & $100 \%$ \\
\hline
\end{tabular}

* La zona de origen de los asociados se ha construido a partir de la pregunta: "¿De qué países de origen son los asociados de su asociación?" Los que tienen solo asociados de países del norte de África (Marruecos, Argelia, Mauritania, Túnez y Egipto) pertenecen a esta categoría (30,1\% de la muestra), los que solo los tienen del resto de países de África se han considerado subsaharianos (36,9\%) y, en tercer lugar, los que tienen asociados de ambos territorios se han ubicado dentro de la categoría mixta (33\%). Concretando más la categoría "mixta", hallamos que no solo se trata de asociaciones que tienen personas del norte de África y subsaharianas, sino que también aparecen en la asociación españoles (lo más frecuente) o ciudadanos europeos, asiáticos y latinoamericanos. Es decir, se trata de asociaciones de africanos en sentido amplio pero que también incorporan a personas de otros orígenes aunque, por cómo se ha elegido el perfil de las asociaciones que se considerarían, la población de estudio (los "no africanos") debe ser de menos del 25\% de los asociados.

Fuente: Elaboración propia a partir de los datos del proyecto CSO2008-01122/SOCl.

más de 45. Regresando al conjunto de los asociados, podemos observar que la media es diferente en función de la comunidad autónoma, de forma que Cataluña tendría las asociaciones con menor número de asociados y Navarra contaría con las más numerosas. También hallamos equivalencias en relación con los años de funcionamiento, de forma que las más jóvenes tienen menos asociados que las más antiguas, en términos relativos. A esto hay que señalar la excepción de las que llevan 20 o más años funcionando, que no cumplen esta relación.

Respecto al número de beneficiarios, es decir, las personas que nuestros entrevistados consideran que se ven beneficiadas por el trabajo que realiza la asociación, siendo conscientes de que este dato es también subjetivo ${ }^{10}$, hallamos un importante incremento

\footnotetext{
${ }^{10}$ Posiblemente se trata de una cifra sobredimensionada con el objetivo de resaltar la importancia de la asociación y el trabajo que realizan.
} 
Tabla 2.

Número de asociados de las asociaciones

\begin{tabular}{|c|c|c|c|c|c|c|c|c|c|}
\hline & & \multicolumn{3}{|c|}{ COMUNIDADAUTÓNOMA } & \multicolumn{5}{|c|}{ AÑOS FUNCIONANDO COMO ASOCIACIÓN } \\
\hline & TOTAL & Cataluña & \begin{tabular}{|l|} 
Comunidad \\
Valenciana
\end{tabular} & Navarra & $\begin{array}{c}\text { Menos } \\
\text { de } 3\end{array}$ & De 4 a 8 & De 9 a 3 & $\begin{array}{c}\text { De } 14 \text { a } \\
19\end{array}$ & Más de 19 \\
\hline Menos de 25 & $8,7 \%$ & $10,9 \%$ & $5,6 \%$ & - & $20,8 \%$ & $6,3 \%$ & $4,7 \%$ & - & $7,7 \%$ \\
\hline De 25 a 50 & $26,7 \%$ & $30,4 \%$ & $20,4 \%$ & $14,3 \%$ & $29,2 \%$ & $34,2 \%$ & $23,3 \%$ & $4,3 \%$ & $23,1 \%$ \\
\hline De 51 a 100 & $25,7 \%$ & $23,2 \%$ & $25,9 \%$ & $50 \%$ & $25 \%$ & $29,1 \%$ & $25,6 \%$ & $26,1 \%$ & $7,7 \%$ \\
\hline Más de 100 & $36,4 \%$ & $33,3 \%$ & $44,4 \%$ & $35,7 \%$ & $22,9 \%$ & $27,8 \%$ & $41,9 \%$ & $69,6 \%$ & $61,5 \%$ \\
\hline $\begin{array}{l}\text { No tienen } \\
\text { socios }\end{array}$ & $0,5 \%$ & - & $1,9 \%$ & - & - & $1,3 \%$ & - & - & - \\
\hline $\begin{array}{l}\text { No sabe / } \\
\text { No contesta }\end{array}$ & $1,9 \%$ & $2,2 \%$ & $1,9 \%$ & - & $2,1 \%$ & $1,3 \%$ & $4,7 \%$ & - & - \\
\hline Total & $100 \%$ & $100 \%$ & $100 \%$ & $100 \%$ & $100 \%$ & $100 \%$ & $100 \%$ & $100 \%$ & $100 \%$ \\
\hline $\begin{array}{l}\text { Media de } \\
\text { asociados }\end{array}$ & 153,4 & 146 & 165,1 & 180,5 & 106,8 & 125,4 & 174 & 291,5 & 180,7 \\
\hline
\end{tabular}

Fuente: Elaboración propia a partir de los datos del proyecto CSO2008-01122/SOCl.

Tabla 3.

Número de beneficiarios

\begin{tabular}{l|c|c|c|c|c|c|c}
\hline & & \multicolumn{3}{|c|}{ COMUNIDADAUTÓNOMA } & \multicolumn{2}{c}{ ZONA DE ORIGEN DE LOS ASOCIADOS } \\
\hline & TOTAL & Cataluña & $\begin{array}{c}\text { Comunidad } \\
\text { Valenciana }\end{array}$ & Navarra & $\begin{array}{c}\text { Norte } \\
\text { Árica }\end{array}$ & Mixta & Subsáhara \\
\hline Menos de 25 & $5,8 \%$ & $7,2 \%$ & $1,9 \%$ & $7,1 \%$ & $8,1 \%$ & $2,9 \%$ & $6,6 \%$ \\
De 25 a 50 & $6,8 \%$ & $7,2 \%$ & $7,4 \%$ & - & $8,1 \%$ & $7,4 \%$ & $5,3 \%$ \\
De 51 a 100 & $15,5 \%$ & $13 \%$ & $18,5 \%$ & $28,6 \%$ & $17,7 \%$ & $10,3 \%$ & $18,4 \%$ \\
Más de 100 & $35,9 \%$ & $21,7 \%$ & $66,7 \%$ & $57,1 \%$ & $38,7 \%$ & $38,2 \%$ & $31,6 \%$ \\
Ns/Ns & $35,9 \%$ & $50,7 \%$ & $5,6 \%$ & $7,1 \%$ & $27,4 \%$ & $41,2 \%$ & $38,2 \%$ \\
Total & $100 \%$ & $100 \%$ & $100 \%$ & $100 \%$ & $100 \%$ & $100 \%$ & $100 \%$ \\
Media de & 679 & 653 & 641 & 969 & 571 & 1.266 & 283 \\
beneficiarios & $679 \%$
\end{tabular}

Fuente: Elaboración propia a partir de los datos del proyecto CSO2008-01122/SOCI. 
de la media: 679 personas. Esto nos permite concluir que nuestros interlocutores consideran que sus acciones tienen un alto impacto social y que existe una importante diversidad en esta valoración del impacto, como se presenta en la tabla 3 y que también veremos al confeccionar la clasificación de asociaciones.

Para finalizar esta descripción básica de las asociaciones, nos referiremos al territorio en el que centran su actividad, que en la mayoría de los casos es de ámbito local. El $65 \%$ centra su actividad en el municipio y el $26,7 \%$ en la comarca en la que se ubica. Por otro lado, el 33,5\% trabaja en su propia autonomía, el 6,8\% trabaja a nivel español, el $11,7 \%$ lo hace en su país de origen y el 1,5\%, en otros países de Europa. Si diferenciamos por comunidades autónomas, Cataluña, en línea con el tamaño antes referido, es la autonomía en la que las asociaciones centran más su trabajo en el municipio $(80,4 \%$, respecto al $38,9 \%$ de la Comunidad Valenciana y el $14,3 \%$ de Navarra. Las asociaciones de estas dos últimas comunidades extienden considerablemente su área de trabajo a toda la autonomía (el $48,1 \%$ de las asociaciones de la Comunidad Valenciana y el $64,3 \%$ de las de Navarra).

\section{Organización y proyección de las asociaciones}

Para conocer la estructura organizativa de las asociaciones, se preguntó sobre los presidentes, la composición de la junta directiva de la asociación, las asambleas y reuniones que se realizaban y la existencia de comisiones de trabajo. Posteriormente, para conocer su proyección externa, nos centramos en la pertenencia a plataformas o foros y redes de trabajo.

El número de presidentes que han tenido las asociaciones varía dependiendo de los años que llevan funcionando; así, más allá de que las más antiguas son las que tienen una media de presidentes más elevada - las que llevan más de catorce años funcionando tienen una media de tres presidentes; las que llevan de nueve a trece años han tenido dos; las que llevan de cuatro a ocho han tenido una media de 1,5 y, finalmente, las que tienen menos de tres años de funcionamiento, un solo presidente-; la media de presidentes del conjunto de la muestra es de 1,8. Esta media es parecida al comparar las diferentes comunidades autónomas pero no idéntica: 1,6 en la Comunidad Valenciana, 1,8 en Cataluña y 2,3 en Navarra. En el caso de Navarra, es destacable que se den más cambios de presidentes aunque no sea la comunidad que tiene las asociaciones más antiguas (la media de años de existencia de sus asociaciones es 7,3 mientras que la de las asociaciones de Valencia es 6,7 y la de Cataluña 8,9). Esto también se observa en la media de tiempo que dura el mandato de los presidentes: 5,1 años en Cataluña; 4,6 en Valencia y 3,9 en Navarra.

Ninguna de las asociaciones dice prescindir de junta directiva, que suele estar formada por una media de ocho personas o algo más alta. A pesar de que se trate de asociaciones de menor tamaño en Cataluña $(8,4)$ que en el resto de comunidades $(7,4$ en Navarra y 7,7 en la Comunidad Valenciana), solo en el $0,5 \%$ de los casos está formada por menos de tres personas y en el $23,8 \%$ de los casos, entre tres y cinco personas. 
La asamblea es la forma común de funcionar de nuestros interlocutores (solo el 8,3\% no ha respondido a la pregunta y nadie ha indicado que no realizan asambleas). Eso sí, el número es desigual. La media, como puede verse en la tabla siguiente, es de 4,87 y es significativamente más alta en Cataluña. Como vemos, el perfil de las asociaciones de Cataluña se presenta como de menor tamaño, con más personas en las juntas directivas, con mandatos presidenciales más largos y con mayor número de asambleas de los asociados; además, son los que llevan más años funcionando; cuestión que nos parece básica. Por su parte, Navarra, en comparación con las otras dos comunidades autónomas, se hallaría en otra situación: mayor número de asociados y beneficiarios, menor número de personas en las juntas, mayor cambio de presidentes y menor número de asambleas.

Por otro lado, algo más de una tercera parte de las asociaciones se organizan en comisiones de trabajo y la existencia o no de ellas se encuentra relacionada también con los años que llevan funcionando (ver tabla 5): a más años funcionando, mayor porcentaje de asociaciones que trabajan mediante comisiones. Diferenciándolas, como venimos haciendo, territorialmente, vemos que el funcionamiento por comisiones es menor en Cataluña en comparación con las otras dos comunidades autónomas; de ahí, posiblemente, el mayor número de asambleas que realizan las asociaciones de Cataluña (recordemos que se trata de la comunidad autónoma con asociaciones de menor tamaño y con juntas directivas de mayor tamaño).

Concretando más, vemos que los que funcionan por comisiones de trabajo tienen una media de reuniones alta $(11,5)$; eso sí, existen diferencias importantes en función de la comunidad autónoma, ya que en Navarra la media de reuniones es mucho más elevada (26) que en la Comunidad Valenciana $(7,7)$ o que en Cataluña $(10,7)$. Las comi-

Tabla 4.

Número de asambleas con asociados en el año 2008

\begin{tabular}{lcccc}
\hline & & \multicolumn{3}{c}{ COMUNIDADAUTÓNOMA } \\
\hline TOTAL & Cataluña & $\begin{array}{c}\text { Comunidad } \\
\text { Valenciana }\end{array}$ & Navarra \\
\hline Solo 1 & $13,6 \%$ & $9,4 \%$ & $24,1 \%$ & $14,3 \%$ \\
De 2 a 3 & $30,6 \%$ & $30,4 \%$ & $25,9 \%$ & $50 \%$ \\
Más de 3 & $51,4 \%$ & $46,3 \%$ & $14,3 \%$ \\
No sabe/no contesta & $8,3 \%$ & $8,7 \%$ & $3,7 \%$ & $21,4 \%$ \\
Total & $100 \%$ & $100 \%$ & $100 \%$ & $100 \%$ \\
Media de asambleas & 4,87 & 5,14 & 4,35 & 4,18 \\
\hline
\end{tabular}

Fuente: Elaboración propia a partir de los datos del proyecto CSO2008-01122/SOCI. 
siones de trabajo existentes son diversas y las más frecuentes son la de cultura, la de educación, la de deporte y las de organización de actividades y acción social. Posteriormente las presentaremos y las relacionaremos con las actividades que realizan (ver tabla 6).

Estas asociaciones no solo cuentan con asociados que se implican en su funcionamiento, sino que las hay que tienen personal contratado para que la asociación funcione ${ }^{11}$. Eso sí, se trata de una minoría, ya que solo el 5,3\% cuenta con este personal. Se trata, en su mayor parte, de asociaciones de la Comunidad Valenciana, en la que representan el $13 \%$, ya que en Cataluña son el 2,9\% y en Navarra no hay ninguna.

Otra de las cuestiones en que quisimos profundizar fue la coordinación interasociativa cuando esta toma forma de pertenencia a plataformas, foros y redes de trabajo, ya que, como indican M. Méndez y F. Mota (2006), un problema del desarrollo asociativo se halla en este tipo de coordinación. A través de la encuesta advertimos que la pertenencia a plataformas o foros es minoritaria, pues solo un $22,3 \%$ dice que se implica en alguna de ellas. De nuevo, se dan diferencias entre comunidades autónomas. Cataluña es la que presenta menos frecuencia $(15,2 \%)$ y Navarra $(42,9 \%$ ) la que más (la Comunidad Valenciana representa un $35,2 \%$ ). Además, observamos que las inscritas en registros y directorios son las que más se implican en plataformas y foros $(26,2 \%)$, mientras que de las no inscritas solo lo hace un $15 \%$.

Tabla 5.

Existencia de comisiones de trabajo en las asociaciones.

\begin{tabular}{l|c|c|c|c|c|c|c|c|c}
\hline & & \multicolumn{3}{|c|}{ COMUNIDAD AUTÓNOMA } & \multicolumn{5}{c}{ AÑO DE CREACIÓN } \\
\hline & TOTAL & Cataluña & $\begin{array}{c}\text { Comunidad } \\
\text { Valenciana }\end{array}$ & Navarra & Menos de 3 & De 4 a 8 & De 9 a 3 & De 14 a 19 & Más de 19 \\
\hline Sí & $35,4 \%$ & $31,2 \%$ & $42,6 \%$ & $50 \%$ & $29,2 \%$ & $30,4 \%$ & $39,5 \%$ & $60,9 \%$ & $30,8 \%$ \\
No & $61,7 \%$ & $65,9 \%$ & $53,7 \%$ & $50 \%$ & $66,7 \%$ & $67,1 \%$ & $60,5 \%$ & $30,4 \%$ & $69,2 \%$ \\
No sabe/ & $2,9 \%$ & $2,9 \%$ & $3,7 \%$ & - & $4,2 \%$ & $2,5 \%$ & - & $8,7 \%$ & - \\
no contesta & $100 \%$ & $100 \%$ & $100 \%$ & $100 \%$ & $100 \%$ & $100 \%$ & $100 \%$ & $100 \%$ & $100 \%$ \\
Total & $100 \%$ &
\end{tabular}

Fuente: Elaboración propia a partir de los datos del proyecto CSO2008-01122/SOCl.

${ }^{11}$ Para M. Méndez y F. Mota (2006), aunque se refieran de forma global a las asociaciones existentes en España: "Uno de los indicadores más claros de profesionalización en el funcionamiento de las asociaciones es la presencia de personal contratado para realizar sus actividades, especialmente de personal pagado a tiempo completo" (p. 213). 
Tabla 6 .

Comisiones de trabajo existentes en las asociaciones que trabajan por comisiones.

\begin{tabular}{l|c|ccc}
\hline & & \multicolumn{3}{|c}{ COMUNIDAD AUTÓNOMA } \\
\hline Base: trabajan por comisiones & TOTAL & Cataluña & $\begin{array}{c}\text { Comunidad } \\
\text { Valenciana }\end{array}$ & Navarra \\
\hline Cultura & $67,1 \%$ & $60,5 \%$ & $78,3 \%$ & $71,4 \%$ \\
Religión & $16,4 \%$ & $16,3 \%$ & $13 \%$ & $28,6 \%$ \\
Educación & $35,6 \%$ & $27,9 \%$ & $43,5 \%$ & $57,1 \%$ \\
Deportes & $26 \%$ & $23,3 \%$ & $34,8 \%$ & $14,3 \%$ \\
Organización de actividades & $21,9 \%$ & $23,3 \%$ & $21,7 \%$ & $14,3 \%$ \\
Acción social & $20,5 \%$ & $14 \%$ & $30,4 \%$ & $28,6 \%$ \\
Comunicación & $16,4 \%$ & $18,6 \%$ & $17,4 \%$ & - \\
Cooperación & $12,3 \%$ & $14 \%$ & $4,3 \%$ & $28,6 \%$ \\
Género & $9,6 \%$ & $11,6 \%$ & $4,3 \%$ & $14,3 \%$ \\
Mediaciónintercultural & $6,8 \%$ & $9,3 \%$ & $4,3 \%$ & - \\
No sabe/no contesta & $1,4 \%$ & - & $4,3 \%$ & - \\
\hline
\end{tabular}

Fuente: Elaboración propia a partir de los datos del proyecto CSO2008-01122/SOCl.

La utilidad real de estas plataformas y foros no está clara entre nuestros entrevistados, como indica la elevada "no respuesta" a esta cuestión: el 61,7\% (aunque, más bien, podríamos decir que no sabían a lo que nos referíamos). Eso sí, pese a que el $16 \%$ dice que no tienen utilidad, un $12,6 \%$ considera que constituyen una mejora en la coordinación, el 10,7\% considera que dotan a la asociación de una mayor proyección social, el 7,3\% que incrementan la influencia de la asociación en las instituciones y el $1,5 \%$ considera que defienden los derechos de los extranjeros representados.

La participación en redes de trabajo también es minoritaria y representa, de nuevo, el $22,3 \%$. Eso sí, con menos diferencias entre comunidades autónomas, aunque las hay ( $21 \%$ en Cataluña; $27,8 \%$ en la Comunidad Valenciana y $14,3 \%$ en Navarra). Existen diferencias entre las registradas o inscritas en directorios (31,1\%) respecto a las que no lo están (11,7\%).

En el caso de las redes, de nuevo vemos que se conoce poco su utilidad ya que el $67 \%$ no sabe o no responde a la pregunta. Por otro lado, el $10,2 \%$ cree que no tiene ninguna; mientras que, los que sí las consideran útiles argumentan que las redes: 
permiten la coordinación de las actuaciones (19,4\%), incrementan la influencia ante las instituciones $(5,3 \%)$, permiten tener más relevancia social $(5,3 \%)$ y permiten defender mejor los derechos de los extranjeros $(0,5 \%)$.

Más allá de que exista poco conocimiento sobre el tema, las respuestas dadas nos indican que, cuando son valorados, los foros, las plataformas y las redes son reconocidos, sobre todo, por lo que representan de mejor coordinación entre los individuos que actúan con la población extranjera o en cuestiones relacionadas con ella y por la visibilidad e influencia (social y política) que permiten a los colectivos. Respecto a esta última cuestión, nos interesamos por conocer cómo retrataban las asociaciones la relación que mantenían con la Administración (aquella/s con la/s que se relacionaban habitualmente) y su respuesta fue que, en la mayoría de casos, se trataba de una relación de colaboración $(87,4 \%)$, aunque también el 10,7\% indicó que era una relación de dependencia o de competencia $(0,5 \%)$. Solo el 7,3\% dijo no tener relación con ellas ${ }^{12}$. Si lo diferenciamos por comunidades autónomas, vemos que en Navarra es donde más se considera que la relación es de dependencia (en un 21,4\%); en Cataluña, en un $12,3 \%$, y en la Comunidad Valenciana en un $3,7 \%{ }^{13}$.

\section{La evolución de las asociaciones: estabilidad e inestabilidad}

Más allá de la trayectoria de las asociaciones que hemos trabajado, para definir su evolución nos interesamos por la estabilidad de las mismas a través del estudio de sus cambios de nombre (desde su creación) y de las altas y bajas de asociados. Los resultados apuntan a que la mayoría de ellas han mantenido el mismo nombre desde su creación $(93,2 \%)$ y solo el $4,9 \%$ dice haberlo modificado ${ }^{14}$. Eso sí, observamos diferencias entre comunidades autónomas. El mantenimiento del nombre es mayor en la comunidad autónoma de Cataluña (solo el 2,9\% lo ha modificado) que en la Comunidad Valenciana $(7,4 \%)$ o en Navarra (14,3\%). Entre los que han realizado este cambio, la mayoría lo ha hecho en una sola ocasión $(90 \%)$ y el resto en dos ocasiones.

\footnotetext{
${ }^{12}$ La dependencia económica, aunque no es la única que se menciona, es la más importante. De hecho, el presupuesto de estas asociaciones se reparte de la siguiente forma (la aportación de los socios es la principal fuente de recursos pero también se recurre a las administraciones): el 60,49\% es aportación de los asociados; el $30,67 \%$, de subvenciones de instituciones españolas; el 3,04\%, subvenciones de fundaciones españolas; el $0,64 \%$, subvenciones de países de origen; $5,16 \%$, otras aportaciones sin concretar. Especificamente, el $51,9 \%$ dice recibir subvenciones de ayuntamientos; el 11,2\%, del Consell Comarcal; el 11,2\%, de la Diputación; el $32 \%$, de gobiernos autonómicos; el 3,9\%, del Gobierno estatal; el 7,3\%, de la Fundación La Caixa; el 5,9\%, de otras fundaciones u obras sociales de bancos y cajas; el 1\%, de la Universidad; etc.

${ }^{13}$ Se expresa más relación de dependencia y, también, de colaboración con las administraciones. Respecto a fundaciones, entidades que trabajan con inmigrantes, asociaciones de inmigrantes, instituciones del país de origen y entidades y asociaciones del país de origen, también aparece la colaboración, pero en menor porcentaje. Además, la idea de competencia aparece fundamentalmente respecto a las citadas entidades y asociaciones del país de origen (argumento citado por el 9,2\% de los encuestados) pero también respecto a las instituciones del país de origen $(4,9 \%)$ o respecto a otras asociaciones de inmigrantes ya en España $(4,9 \%)$.

${ }^{14} \mathrm{El} 1,9 \%$ no responde a la pregunta.
} 
Otro indicador del grado de estabilidad/inestabilidad de las asociaciones nos lo dan las altas y bajas de los asociados. Al preguntar sobre la frecuencia de estos movimientos, un $5 \%$ nos dice que son muy frecuentes; un $21,8 \%$, que lo son bastante; un $41,5 \%$, que son poco frecuentes y el $30,7 \%$, que no son nada frecuentes (el restante $1 \%$ no responde). La media resultante de otorgar valor a cada una de las citadas respuestas ${ }^{15}$ se sitúa en el "poco frecuente" (media de 2,01). No obstante, al diferenciarlo por comunidades autónomas, observamos que las asociaciones más "inestables" serían las navarras (más cercanas al "bastante frecuente", ya que el $43 \%$ se sitúa en esta franja y el $14,3 \%$ en el "muy frecuente"), en comparación con las otras dos comunidades. Todo ello redunda en el hecho de que Navarra cuenta con asociaciones más jóvenes, más inestables.

Todo lo anterior se complementa con una pregunta para conocer el porcentaje de bajas y altas del último año (2008). La media de bajas fue del $9,25 \%$ de los asociados y la de altas del $15,4 \%$. De nuevo, Navarra fue la que tuvo mayor inestabilidad, ya que su media de bajas fue del $29,8 \%$ de los asociados y la de altas del $25,5 \%$. En cambio, en Cataluña fue del $8,4 \%$ y $14,2 \%$ y en la Comunidad Valenciana del 6,3 y $13,8 \%$, respectivamente.

\section{Las asociaciones de inmigrantes africanos. Ensayo tipológico}

Como hemos visto, en función de las variables que hemos tenido en cuenta, se detectan perfiles diferenciados entre las asociaciones de las tres comunidades autónomas pero la explicación del fenómeno todavía nos pareció limitada. Por ello, realizamos ensayos tipológicos que nos permitieron una mejor comprensión de la fotografía de estas asociaciones. Utilizando el análisis de clúster ${ }^{16} \mathrm{y}$ realizando diferentes ensayos con variables definitorias de la estructura y organización de las asociaciones, fuimos detectando las que representaban la tipología más explicativa de todas ellas. Finalmente, han resultado, según criterios estadísticos y de capacidad explicativa [Fernández Santana (1991)], cuatro grupos sociales (considerados según su proximidad intraclúster y lejanía interclúster).

Como hemos indicado, se han realizado diversos ensayos utilizando diferentes variables obtenidas a través del cuestionario, algunas de ellas fueron eliminadas por no ser explicativas de las diferencias entre las asociaciones en lo que se refiere a estructura y organización. Las variables que finalmente fueron utilizadas son: la existencia o no de comisiones de trabajo, el número de asambleas que realizan anual-

\footnotetext{
${ }^{15}$ La respuesta "muy frecuente" equivale al valor 4; "bastante frecuente" a 3; "poco frecuente" a 2, y "nada frecuente" a 1.

${ }^{16}$ Se utilizó el programa de análisis Cluster Galaxy del programa STAR que, básicamente, clasifica las observaciones en grupos próximos entre sí y distantes del resto de grupos. Es un análisis tipológico no jerárquico y el método es iterativo heurístico.
} 
mente (en las que se convoca al conjunto de los asociados), la implicación en foros o plataformas de coordinación e intercambio de opiniones, la pertenencia a redes de trabajo y la existencia y el número de personas contratadas que trabajan en la asociación. Estas fueron las variables que resultaron más definitorias para confeccionar una tipología que explicaba el $65 \%$ de la varianza y que nos proporcionaba una visión interesante de la diferente estructura y forma de organización y trabajo de las asociaciones. A continuación presentamos la tipología resultante:

- Profesionalizadas ${ }^{17}$ : se trata de asociaciones que están consolidadas; de hecho, ha habido ocasiones en que nos han parecido casi una empresa. Se trata de una minoría dentro de la muestra. Concretamente, estamos hablando de once asociaciones (el 5,3\% de las encuestadas) que se definen principalmente por tener un número alto, en términos relativos, de personal contratado; así como por el hecho de tener más proyección exterior (en comparación con buena parte de los otros clústers), trabajar en red, disponer de comisiones de trabajo y no realizar muchas asambleas de todos los asociados. Se trata de asociaciones que tienen un grado mayor de organización, a nivel de estructura y de trabajo. Podemos completar el perfil de este tipo de asociaciones a partir de la segmentación de todas las preguntas de la encuesta con la tipología obtenida. De esta forma, en comparación con las otras, estas asociaciones son las que llevan más años funcionando (una media de 10,7) y cuentan con estatutos elaborados (9,6 años de media). Además, se trata de asociaciones que tienen un alto número de socios (319 de media) y de beneficiarios (2607). En último lugar, hay que decir que se trata de asociaciones que trabajan en un territorio amplio y, además, diversifican sus fuentes de ingresos.

- Voluntaristas de base: para diferenciar las restantes tipologías de la anterior, más profesionalizada, a las siguientes las hemos definido primeramente como voluntaristas, ya que en gran medida basan su funcionamiento en el voluntariado. En el caso concreto de las voluntaristas de base, además de que el voluntariado sea la forma habitual de trabajo en ellas, las define el hecho de que existe poca organización en su forma de funcionamiento. Se definen por ser las que trabajan menos en comisiones de trabajo y, poco, en foros, plataformas y redes; en que apenas tienen personal contratado; y en que en lo único que destacan levemente, y en positivo, es en la realización de asambleas. De ahí que las llamemos "de base", entendiéndolo como el primer estadio del asociacionismo, pues en ellas no existe una organización clara de

\footnotetext{
${ }^{17}$ Los nombres dados a cada tipología, a pesar de no ser fáciles de etiquetar, han sido discutidos y decididos por todo el equipo de investigación y se pretende que identifiquen lo más relevante de cada una de ellas, es decir, lo que une intragrupo y qué diferencias hay intergrupos.
} 
la estructura y de sus funciones. Es destacable el hecho de que se trata de la tipología más numerosa: 95 asociaciones pertenecen a esta tipología, es decir, el $46,15 \%$ del total. Completamos el perfil de este tipo de asociaciones a partir del análisis del resto de datos de la encuesta y detectamos que se trata de las que llevan menos años funcionando (una media de 6,5) y las que han estado menos tiempo rigiéndose por unos estatutos (6,1 años de media); son asociaciones que tienen un número menor de socios (125 de media) aunque no de beneficiarios (cuyo número asciende a 459). En último lugar, hay que destacar que se trata de asociaciones que trabajan en un territorio muy concreto y, además, sus fuentes de ingresos provienen principalmente de las aportaciones de los asociados.

- Voluntaristas organizadas: se trata de asociaciones que han realizado algunos pasos orientados a organizarse interiormente y formalmente. Se distinguen del resto, especialmente, por el hecho de que trabajan habitualmente en comisiones de trabajo. Por lo que respecta a las otras variables (realización de asambleas de todos los asociados, pertenencia a foros, plataformas y redes, o a tener o no personal contratado), se definen en negativo. En este caso, se trata de 57 asociaciones, es decir, el $27,65 \%$ de las asociaciones encuestadas. Completamos este perfil a partir del análisis del resto de la encuesta y detectamos que se trata de asociaciones que llevan algunos años más funcionando que las asociaciones de base (una media de 8,9 ) y que cuentan con estatutos ( 8,6 años de media). Se trata de asociaciones que tienen un número mayor de socios que las de base (169 de media), aunque no de beneficiarios (351), ya que, recordemos, están más centradas en sí mismas. En último lugar, destacamos que se trata de asociaciones que trabajan en un territorio algo más amplio que las anteriores y, además, aunque sus fuentes de ingresos llegan principalmente de las aportaciones de los asociados, las diversifican algo más mediante subvenciones.

- Voluntaristas reticulares: se trata de asociaciones que, como la anterior, también han realizado pasos hacia una mayor organización, en comparación con las voluntaristas de base, pero su enfoque ha sido más exterior que interior. Se distinguen especialmente por su labor en foros, plataformas y redes, sin destacar, en comparación con el resto, por el trabajo en comisiones y la realización de asambleas de todos los asociados y, eso sí, siguen, como el resto de asociaciones voluntaristas, sin definirse por tener personal contratado. En este caso, se trata de 43 asociaciones, es decir, el $20,9 \%$ de la muestra. Si completamos el perfil de este tipo de asociaciones a partir del análisis del resto de la encuesta, detectamos que son, de entre las voluntaristas, las que llevan más años funcionando (una media de 10,2 ) pero que no llegan a la veteranía de las profesionalizadas aunque sí cuentan con estatutos (nueve años 
de media). Por otro lado, no son asociaciones que tengan un número mucho mayor de socios que las voluntaristas organizadas (154 de media) pero sí lo tienen de beneficiarios (668), debido al énfasis que ponen en la proyección exterior. En último lugar, cabe mencionar que se trata de asociaciones que diversifican su territorio de trabajo y que, además, actúan en sus países de origen. Sus fuentes de ingresos son, principalmente, las aportaciones de los asociados aunque también acuden a subvenciones (especialmente de ayuntamientos), por ejemplo, para la cooperación internacional.

Las anteriores tipologías no se distribuyen territorialmente de forma homogénea. La Comunidad Valenciana presenta una distribución más equilibrada que el resto de comunidades: el $13 \%$ de sus asociaciones pertenecen a las profesionalizadas; el 33,3\% voluntaristas de base; el 25,9\% voluntaristas estructuradas y el $27,8 \%$ voluntaristas reticulares. Por su parte, en Cataluña, el 2,9\% son asociaciones profesionalizadas; el $50,7 \%$ voluntaristas de base; el $27,5 \%$ voluntaristas estructuradas y el $18,8 \%$ voluntaristas reticulares. Por lo que respecta a Navarra, no hallamos asociaciones profesionalizadas; el $50 \%$ son voluntaristas de base; el $35,7 \%$ voluntaristas estructuradas y el $14,3 \%$ voluntaristas reticulares.

\section{Objetivos y actuaciones de LAS ASOCIACIONES}

Para finalizar, nos centraremos en presentar los objetivos y las actividades que realizan las asociaciones, relacionándolos con las tipologías resultantes, además de con otras varibles básicas que hemos ido considerando.

Los principales objetivos de las asociaciones en el momento de realizar la entrevista eran $^{18:}$ : el mantenimiento de la cultura de origen $(53,4 \%)$, el apoyo legal a los inmigrados $(46,1 \%)$, el apoyo laboral a los inmigrados $(40,3 \%)$, el apoyo a los inmigrados desde el punto de vista residencial $(33,5 \%)$, la creación de un espacio de encuentro (32\%), el fomento de la convivencia $(15,5 \%)$, el mantenimiento de la religión $(14,1 \%)$, la acogida $(12,1 \%)$, la ayuda a los países de origen $(10,2 \%)$, la difusión de las lenguas/culturas autóctonas a otros inmigrados $(9,7 \%)$ y la formación de los adultos (5,3\%). Vemos, pues, que se trata de objetivos sociales y culturales, y que apenas son mencionados los objetivos políticos.

En relación con los anteriores objetivos, las principales actividades realizadas por las asociaciones son: la mediación (43,2\%), la organización de sesiones de sensibilización externa $(30,6 \%)$, los proyectos de codesarrollo $(26,7 \%)$, los proyectos de inserción labo-

\footnotetext{
${ }^{18}$ La pregunta se realizó sin condicionar ningún tipo de respuesta, solo preguntando por los objetivos de su asociación.
} 
ral $(22,8 \%)$ y la impartición de clases de lengua española para mujeres $(22,8 \%)$ y hombres $(21,4 \%)$. De esta forma, hallamos actividades intragrupo, actividades extragrupo y actividades de mediación, y, aunque no en igual porcentaje, todas ellas con una importante presencia. Las actividades intragrupo están enfocadas a la inserción laboral, económica y cultural (como la enseñanza de lenguas oficiales y de lenguas de origen); las extragrupo están más centradas en la sensibilización y el codesarrollo, y la mediación, en proyectos de mediación intercultural [para ampliar, véase Llevot y Garreta (2013)].

Al preguntar sobre otras actividades que se habrían querido organizar en las asociaciones pero sin posibilidad de llevarlas a cabo, se repiten las respuestas: un 13,1\% de las asociaciones habría querido organizar formación en lengua castellana para mujeres y un $12,1 \%$, para hombres; por lo que respecta a la lengua catalana, un $11,7 \%$ habría querido cursos de formación para mujeres y un $11,2 \%$, para hombres; el $6,8 \%$ habría querido realizar proyectos de inserción laboral; el $6,8 \%$, proyectos de codesarrollo; el $4,9 \%$, de mediación y el $3,9 \%$, proyectos de acogida. El motivo de no haber realizado estas actividades es, sobre todo, económico (la falta de dinero ha sido el argumento dado por el $85,8 \%$ de los entrevistados). Además, el 20,6\% indica que la falta de personal es también un motivo y el 6,4\% aduce como explicación la falta de un local para realizarlas. Al tener en cuenta los cuatro clústers construidos, detectamos que la falta de dinero es un problema menor para las asociaciones más profesionalizadas (el $66,7 \%$ ), respecto a lo que esta falta supone para las voluntaristas reticulares (pues afecta al $79,5 \%$ ), las voluntaristas de base (al $86,2 \%$ ) y las voluntaristas estructuradas (al $94,7 \%$ ). Por otro lado, la falta de personal parece que también es un problema menor para las profesionalizadas (lo es para el $16,7 \%$ de ellas), mientras que es un problema grave para el $20 \%$ de las reticulares, el $20,7 \%$ de las de base y el $21,1 \%$ de las estructuradas. En último lugar, cabe mencionar que las profesionalizadas se diferencian por aportar una respuesta inexistente entre las otras tipologías: el 16,7\% de ellas dice que "no tiene capacidad para organizar más cosas".

Todo lo anterior tiene que ver con las "principales necesidades que tienen las asociaciones"; el $88,3 \%$ nos indica que se trata de necesidades económicas; el $54,9 \%$, de infraestructuras (locales); el 22,3\%, de mejora de la relación con las instrucciones; el $15,5 \%$, de apoyo en la implementación de proyectos; el 7,8\%, de apoyo para el diseño de proyectos y el $5,3 \%$, de mejora de la coordinación con otras asociaciones de personas de origen inmigrado. De forma breve, los problemas económicos son menores, aunque existen y son la respuesta más citada, entre las asociaciones profesionalizadas (81,8\%), mientras que sí lo son para un $86 \%$ de las voluntaristas estructuradas, un $88,4 \%$ de las voluntaristas reticulares y el $90,5 \%$ de las voluntaristas de base. Para el $9,1 \%$ de las profesionalizadas, la colaboración con las instituciones tampoco es un problema tan importante, aunque sí lo es para el $31,6 \%$ de las estructuradas, el $23,3 \%$ de las reticulares y el $17,9 \%$ de las de base. Finalmente, destacamos que las profesionalizadas inciden más que el resto en el hecho de que que necesitarían más apoyo para diseñar proyectos $(27,3 \%)$ y para implementarlos (18,2\%) aunque, respecto a esta última cuestión, son las asociaciones reticulares las que más lo mencionan $(44,2 \%)$. 
Tabla 7.

Principales actividades de su asociación.

\begin{tabular}{|c|c|c|c|c|c|}
\hline & TOTAL & Profesionalizadas & $\begin{array}{c}\text { Voluntaristas } \\
\text { de base }\end{array}$ & $\begin{array}{l}\text { Voluntaristas } \\
\text { estructuradas }\end{array}$ & $\begin{array}{c}\text { Voluntaristas } \\
\text { reticulares }\end{array}$ \\
\hline Formación lengua castellana para hombres & $21,4 \%$ & $54,5 \%$ & $16,8 \%$ & $24,6 \%$ & $18,6 \%$ \\
\hline Formación lengua castellana para mujeres & $22,8 \%$ & $63,6 \%$ & $16,8 \%$ & $26,3 \%$ & $20,9 \%$ \\
\hline Formación lengua catalana para hombres & $15 \%$ & $9,1 \%$ & $15,8 \%$ & $24,6 \%$ & $2,3 \%$ \\
\hline Formación lengua catalana para mujeres & $16 \%$ & $9,1 \%$ & $15,8 \%$ & $24,6 \%$ & $7 \%$ \\
\hline Formación lengua valenciana para hombres & $2,4 \%$ & - & $2,1 \%$ & $3,5 \%$ & $2,3 \%$ \\
\hline Formación lengua valenciana para mujeres & $2,9 \%$ & - & $2,1 \%$ & $5,3 \%$ & $2,3 \%$ \\
\hline Formación lengua euskera para mujeres & $0,5 \%$ & $9,1 \%$ & - & - & - \\
\hline Formación lengua castellana para niños & $10,2 \%$ & $27,3 \%$ & $7,4 \%$ & $15,8 \%$ & $4,7 \%$ \\
\hline Formación lengua castellana para niñas & $10,2 \%$ & $27,3 \%$ & $7,4 \%$ & $15,8 \%$ & $4,7 \%$ \\
\hline Formación lengua catalana para niños & $5,8 \%$ & - & $7,4 \%$ & $8,8 \%$ & - \\
\hline Formación lengua catalana para niñas & $5,8 \%$ & - & $7,4 \%$ & $8,8 \%$ & - \\
\hline Formación lengua valenciana para niños & $2,4 \%$ & - & $2,1 \%$ & $3,5 \%$ & $2,3 \%$ \\
\hline Formación lengua valenciana para niñas & $2,4 \%$ & - & $2,1 \%$ & $3,5 \%$ & $2,3 \%$ \\
\hline Clases de religión & $8,3 \%$ & $9,1 \%$ & $6,3 \%$ & $14 \%$ & $4,7 \%$ \\
\hline Organizar sesiones sensibilización externa & $30,6 \%$ & $63,6 \%$ & $21,1 \%$ & $21,1 \%$ & $55,8 \%$ \\
\hline Proyectos de mediación & $43,2 \%$ & $45,5 \%$ & $37,9 \%$ & $38,6 \%$ & $60,5 \%$ \\
\hline Proyectos de inserción laboral & $22,8 \%$ & $45,5 \%$ & $17,9 \%$ & $24,6 \%$ & $25,6 \%$ \\
\hline Proyectos de codesarrollo & $26,7 \%$ & $45,5 \%$ & $12,6 \%$ & $42,1 \%$ & $32,6 \%$ \\
\hline Acogida & $9,2 \%$ & $9,1 \%$ & $11,6 \%$ & $8,8 \%$ & $4,7 \%$ \\
\hline Actividades de relación para mujeres inmigradas & $1 \%$ & - & $1,1 \%$ & - & $2,3 \%$ \\
\hline Actividades de formación para mujeres inmigradas & $1,9 \%$ & - & $1,1 \%$ & $3,5 \%$ & $2,3 \%$ \\
\hline Ayuda países de origen & $2,4 \%$ & - & $4,2 \%$ & $1,8 \%$ & - \\
\hline Ayuda económica a inmigrantes necesitados & $1,5 \%$ & - & - & $5,3 \%$ & - \\
\hline Asesoramiento legal & $1,9 \%$ & $9,1 \%$ & $3,2 \%$ & - & - \\
\hline Clases de lengua de origen & $11,7 \%$ & $27,3 \%$ & $11,6 \%$ & $15,8 \%$ & $2,3 \%$ \\
\hline Clases de cultura de origen & $10,7 \%$ & $27,3 \%$ & $6,3 \%$ & $19,3 \%$ & $4,7 \%$ \\
\hline Cursos de informática & $3,4 \%$ & $18,2 \%$ & $2,1 \%$ & $3,5 \%$ & $2,3 \%$ \\
\hline Otros cursos/talleres que interesan a asociados & $9,2 \%$ & $45,5 \%$ & $4,2 \%$ & $10,5 \%$ & $9,3 \%$ \\
\hline Fiestas & $9,2 \%$ & - & $11,6 \%$ & $14 \%$ & - \\
\hline Actividades deportivas & $2,9 \%$ & - & $3,2 \%$ & $1,8 \%$ & $4,7 \%$ \\
\hline Otras & $22,8 \%$ & $54,5 \%$ & $22,1 \%$ & $19,3 \%$ & $20,9 \%$ \\
\hline No sabe/no contesta & $2,4 \%$ & - & $5,3 \%$ & - & - \\
\hline
\end{tabular}

Fuente: Elaboración propia a partir de los datos del proyecto CSO2008-01122/SOCl. 


\section{CONCLUSIONES}

Las asociaciones de inmigrantes procedentes de países de África formadas en las comunidades autónomas de Cataluña, Valencia y Navarra son jóvenes ${ }^{19}$ (aunque en distinto grado) ya que se han originado a partir de una inmigración reciente. Además, partiendo de sus objetivos y actividades, es razonable concluir que estas asociaciones no se encuentran aisladas de su entorno; al contrario, todas ellas tienen una doble función: hacer frente a las necesidades internas de sus miembros e interactuar con su contexto exterior. En línea con el trabajo de J. Nyhagen (2008), hemos visto que la mayoría de las asociaciones no tratan de influir en los asuntos políticos; de hecho, no domina en ellas un objetivo político [aunque, al pertenecer a foros, redes y plataformas, en parte, lo están haciendo, y, profundizando a través de las entrevistas, puede detectarse que también existen estos objetivos; véase al respecto el trabajo de Moncusí y Albert (2013)] sino más bien sociocultural. Sobre su impacto en el entorno, según la opinión de nuestros interlocutores, las acciones que realizan tienen un alto impacto social (por los beneficiarios que mencionan, las relaciones que tienen con la Administración, entidades, etc.), aunque existe una importante diversidad entre ellas (en función de los años que llevan funcionando, sus objetivos y su posición en la tipología que hemos presentado). En consonancia con la hipótesis de trabajo, una de las características de este asociacionismo es su diversidad, de estructuras organizativas y de funcionamiento, que guarda relación con los años de existencia de la asociación. Observamos cómo el tiempo favorece su consolidación y mayor estructuración, aunque en ello también influyen factores como las dinámicas de liderazgo, la existencia o no de conflictos internos, o el mayor o menor apoyo externo recibido (Garreta 2012).

Con el tiempo, existe la tendencia a una mayor organización interna y externa de las asociaciones pero no se puede concluir que la dinámica de todas ellas sea evolucionar hacia modelos asociativos más estructurados y profesionalizados. Además de las entrevistas en profundidad, que en este artículo no presentamos, del proyecto de investigación ${ }^{20}$ se puede concluir que, con el paso del tiempo, en muchos casos se produce una evolución en el citado sentido de disponer de una mayor organización y estructuración de las asociaciones. Aunque esto no quiere decir que el objetivo de todas ellas sea convertirse en asociaciones profesionalizadas, ya que para algunas sus objetivos pueden realizarse, e incluso realizarse de mejor manera, manteniéndose como asociaciones voluntaristas de base menos condicionadas por el entorno y, sobre todo, por las instituciones. Al mismo tiempo, hemos visto que las hay que querrían profesionalizar sus dinámicas internas pero no lo han logrado. De hecho, como mostramos hace unos años (Garreta 1998; 2003), la dinámica de las asociaciones se halla influenciada por múltiples factores internos y externos que

\footnotetext{
${ }^{19}$ Hay que tener en cuenta que el asociacionismo en España ya se considera un fenómeno reciente (Méndez y Mota 2006).

${ }^{20}$ Además de en otros artículos del monográfico de Revista Internacional de Sociología en el que se encuentra este artículo, las entrevistas en profundidad han sido analizadas en J. Garreta (2011 y 2012).
} 
las reorientan y reconstruyen (cuando no las hacen desaparecer, fisionar o fusionar con otras); de ahí que, aunque observamos una tendencia a la mayor organización interna y/o proyección externa, a medida que pasan los años, los objetivos y las situaciones con las que se van encontrando van modificando lo que son y lo que hacen.

Al diferenciarlas por comunidades autónomas, observamos que la situación no es homogénea. El perfil de las asociaciones de Cataluña es de menor tamaño, con más personas en las juntas directivas, con mandatos presidenciales más largos, con mayor número de asambleas de los asociados y con más años de funcionamiento, lo que dibuja un asociacionismo más atomizado y consolidado. Por otro lado, la Comunidad Valenciana ha desarrollado un asociacionismo diverso, en el que se observa más equilibrio entre las asociaciones, desde el punto de vista de la tipología, y que estas tienen una duración y situación media. Por su parte, Navarra se hallaría en el otro extremo: mayor número de asociados y beneficiarios, menor número de personas en las juntas, mayor cambio de presidentes y un menor número de asambleas. Como indicamos, esta diferencia se explica por los años de funcionamiento de las asociaciones pero también creemos, aunque aquí no ha sido analizado, que el entorno político (y social) puede ayudar a explicar los diversos desarrollos y consolidaciones que han seguido.

En síntesis, nuestros datos evidencian que nos encontramos con asociaciones de inmigrantes jóvenes que persiguen objetivos eminentemente socioculturales, se encuentran en etapas de desarrollo diferente (a nivel organizativo) y que siguen desarrollos diferentes según el territorio. La imagen que nos aporta la encuesta es útil para definir una situación concreta y detectar variables importantes en el desarrollo del asociacionismo. Además del tiempo y las dinámicas internas, hay que tener en cuenta las externas (políticas de la Administración e instituciones y otras entidades y asociaciones) y, a partir de ahora, y de un modo especial, su forma de financiación. En este sentido, es importante tener en cuenta que algunas asociaciones han basado parte de su desarrollo en la financiación externa. La actual situación de crisis económica está suponiendo la reducción de recursos disponibles, así como la reorientación de los criterios de gestión. Desde este punto de vista, resultará interesante seguir analizando la evolución de este asociacionismo y ver cómo los factores internos y externos van afectando a su desarrollo y consolidación.

\section{RefERENCIAS BibliográficAS}

Amengual, A. et al. 2003. Participació i immigració en contextos pluriculturals. La situació del casc antic de Barcelona. Barcelona: Fundació Jaume Bofill.

Aparicio, R. y A. Tornos. 2010. Las asociaciones de inmigrantes en España. Una visión de conjunto. Madrid: Observatorio Permanente de la Inmigración del Ministerio de Trabajo e Inmigración.

Aubarell, G. 2003. Gestionar la diversitat. Barcelona: lemed.

Casey, J. 1995. "El papel de las organizaciones no gubernamentales en la elaboración de las políticas públicas: el caso de la integración de inmigrantes extranjeros en Cataluña." Tesis doctoral. Universidad Autónoma de Barcelona, Barcelona (sin publicar). 
Castles, S. y M. J. Miller. 1994. The age of migration. International population movements in the modern world. Londres: The Macmillan Press Ltd.

Colectivo IOE. 1992. La inmigración extranjera en Catalunya. Barcelona: Institut d’Estudis Mediterranis.

Colectivo IOE. 1994. "La inmigración extranjera en España: sus características diferenciales en el contexto europeo." Pp. 83-119 en Los retos de la inmigración. Racismo y pluriculturalidad, editado por J. Contreras. Madrid: Talasa.

Colectivo IOE. 1999. "Espagne: un nouveau pays d'immigration." Migrations Société Vol. 11, n 65 : 63-79.

Cordero-Guzmán, H. 2005. "Community-Based Organisations and Migration in New York City." Journal of Ethnic and Migrations Studies 31: 889-909.

Crespo, R. 1997. "Asociacionismo inmigrante." Pp. 321-354 en II Informe sobre la inmigración y trabajo social. Barcelona: Diputació de Barcelona.

Diani, M. 2000. "Simmel to Rokkan and beyond: towards a network theory of (new) social movements." European Journal of Social Theory 3: 387-406.

Fennema, M. y J. N. Tillie. 2004. "Do immigrant policies matter? Ethnic civic communities and immigrant policies in Amsterdam, Liège and Zurich". Pp. 39-56 in Citizenship in European Cities. Immigrants, Local Politics and Integration Policies, edited by R. Penninx, K. Kraal, M. Martiniello y S. Vertovec. Aldershot: Ashgate.

Fernández Santana, O. 1991. "El análisis de cluster: aplicación, interpretación y validación." Papers. Revista de Sociología 37: 65-76.

Garreta, J. 1998. "Minories ètniques, associacionisme i integració sociocultural." Papers. Revista de Sociología 56: 197-230.

Garreta, J. 2003. La integración sociocultural de las minorías étnicas. Barcelona: Anthropos.

Garreta, J. 2009. Sociedad multicultural e integración de los inmigrantes en Cataluña. Discursos y prácticas. Lleida: Universitat de Lleida.

Garreta, J. 2011. "L'immigration et les politiques d'intégration en Catalogne: quelques enjeux." Migrations Société 23: 139-151.

Garreta, J. 2012. "Asociacionismo e inmigración. Los papeles de las asociaciones de inmigrantes en España." Ponencia presentada en el Congreso de Migraciones Internacionales en España, 13 de abril, Bilbao.

Giddens, A. 1991. Modernity and Self-Identity. Cambridge: University Press.

Gómez Gil, C. 2006. Inmigración y asociacionismo. El caso de Alicante. Alacant: Universitat d'Alacant.

Ibarra, P. y B. Tejerina. 1998. Los movimientos sociales. Transformaciones políticas y cambio cultural. Madrid: Trotta.

Jaussaud, E. 1998. "Experiences of immigrant associations and the emergence of local economic development: the case of the Association Migrations et Développement." Migrations Société 10: 77-85.

Jenkins, S. 1988. Ethnic Associations and the Welfare State: Services to Immigrants in five countries. Nueva York: Columbia University Press. 
Kastoryano, R. 1994. "Construction de communautés et négociation des identités: les migrants musulmans en France et en Allemagne." Pp. 229-244 en Cartes d'identité. Comment dit-on "nous" en politique?, editado por D. C. Martin. París: Presses Fondation Nationale des Sciences Politiques.

Lacomba, J. 2004. "Migración y desarrollo rural en Marruecos. El papel de los emigrantes y sus asociaciones." Pp. 185-212 en Migración y desarrollo, editado por A. Escrivá y N. Ribas. Córdoba: CSIC.

Lacomba, J. y J. Giner. 2013. "La participación en asociaciones de los inmigrantes africanos. Vías de interpretación del distanciamiento asociativo." Revista Internacional de Sociología, Vol. 71, Extra 1:67-89.

Llevot, N. y J. Garreta. 2013. "La mediación intercultural en las asociaciones de inmigrantes de origen africano." Revista Internacional de Sociología, Vol. 71, Extra 1: 167-188.

Lluch, A. 2000. "L'associacionisme dels immigrants." DCIDOB: 67-68.

Mafia, M. M., S. Ballina y P. C. Monkevicius. 2005. "The associations of immigrant foreigners and their descendants in the province of Buenos Aires." Studi Emigrazione 42: 625-642.

Martín, G. 2004. Marroquies en España. Estudio sobre su integración. Madrid: Fundación Repsol YPF.

Martínez, M. F. 2001. "Una tipología analítica de las redes de apoyo social en inmigrantes africanos en Andalucía." REIS vol. 95 n 1: 99-125.

Mata, A. 2011. "Asociacionismo inmigrante y administración local. Relaciones e interdependencias". Ponencia presentada en III Congreso de la Red Española de Política Social, 24-26 de noviembre, Pamplona. (www1.unavarra.es/digitalAssets/155/155457_3.1_Mata_Asociacionismolnmigrante.pdf).

Méndez, M. y F. Mota. 2006. "Las características organizativas de las asociaciones en España." Pp 203-22 en Ciudadanos, asociaciones y participación en España, editado por J. R. Montero, J. Font y M. Torcal. Madrid: CIS.

Moncusí, A. y M. Albert. 2013. "El rol del asociacionismo de inmigrantes africanos en la construcción de cohesión social y la convivencia en Cataluña, Navarra y la Comunidad Valenciana. Miradas cruzadas." Revista Internacional de Sociología, Vol. 71, Extra 1: 39-65.

Morell, A. 2005. "El papel de las asociaciones de inmigrantes en la sociedad de acogida: cuestiones teóricas y evidencia empírica." Migraciones 17: 11-142.

Moya, J. C. 2005. "Immigrants and Associations: a Global and Historical Perspectiva." Journal of Ethnic and Migration Studies 31: 833-864.

Nyhagen, J. 2008. "Political and Cultural Ethnic Mobilisation: The Role of Immigrant Associations in Norway." Journal of Ethnic and Migrations Studies 34: 935-954.

Ozcurumez, S. 2009. "Immigrant Associations in Canada: Included, Accommodated, or Excluded?." Turkish Studies 10: 195-215.

Palidda, S. 2005. "The Italian Association in France." Studi Emigrazione 42: 919-934.

Pilati, K. 2012. "Network Resources and the Political Engagement of Migrant Organisations in Milan." Journal of Ethnic and Migration Studies 38: 671-688.

Poinsot, M. 2000. "Associations de l'immigration et mouvement associatif français." Migrations Société 12: $45-54$. 
Pont, J. 2005. "Aproximación al asociacionismo de inmigrantes extracomunitarios a partir de las teorías de la estructuración". Pp. 201-234 en Integraciones diferenciadas: migraciones en Cataluña, Galicia y Andalucía, editado por C. Solé, C. y A. Izquierdo. Barcelona: Anthropos.

Aubarell, G. (Dir.) 2003. Gestionar la diversitat. Barcelona: lemed.

Schrover, M. y F. Vermeulen. 2005. "Immigrant organisations." Journal of Ethnic and Migration Studies 31: 823-832.

Simó, C., M. Jabbaz, F. Torres, J. Giner y B. Herzog. 2005. "Asociacionismo y población extranjera en la Comunidad Valenciana." Cuadernos electrónicos de filosofía del derecho 12: 1-57.

(De) Singly, F. 1996. Le soi, le couple et la famille. París: Harmattan.

Sipi, R. 2000. "Las asociaciones de mujeres ¿agentes de integración social?." Papers. Revista de Sociología 60: 355-364.

Veredas, S. 1999. "Las asociaciones de inmigrantes marroquíes y peruanos en la Comunidad de Madrid." Tesis doctoral. UCM, Madrid.

JoRd GARRETA Bochaca es profesor titular de sociología del Departamento de Geografía y Sociología de la Universidad de Lérida. Su trabajo investigador se ha centrado en sociología de la educación (escolarización del alumnado de origen extranjero, políticas de integración y participación de las familias en la escuela) y en sociología de las migraciones (movimientos migratorios, integración, participación social). En los últimos años ha sido investigador invitado en l'Université de Montréal y en l'Ecole des Hautes Études en Sciences Sociales (París).

NúRIa Llevot Calvet es profesora contratada doctora de pedagogía en el Departamento de Pedagogía y psicología de la Universidad de Lérida. Su trabajo investigador se ha centrado en mediación intercultural, diversidad cultural y educación y la integración de minorías étnicas. En los últimos años ha sido investigadora invitada en la Université de Montréal, Roma Tre y París 8.

RECIBIDO: 01/09/2012

ACEPTADO: 24/03/2013 